\title{
FUZZY MATHEMATICAL MODEL FOR DESIGNING CYBERSECURITY MANAGEMENT BODIES IN STRATEGIC PLANNING
}

\author{
Dragan Bojanić1, *, \\ Vladimir Ristić ${ }^{2}$ \\ Jan Marček ${ }^{3}$
}

\author{
${ }^{1}$ Strategic Research Institute, \\ University of Defence in Belgrade, \\ Belgrade, Serbia \\ 2Military Academy, \\ University of Defence in Belgrade, \\ Belgrade, Serbia \\ ${ }^{3}$ Faculty for Business Studies and Law, \\ University „Union Nikola Tesla”, \\ Belgrade, Serbia
}

\begin{abstract}
:
It is necessary for the design of organizational structure of cybersecurity systems or information security systems to meet the basic corporate goals and objectives. Thus, although cyber security is only an element of information security, it is its most important part. For that reason, it is essential for the organizational structure to be constantly perfected. The analysis of strengths, weaknesses, opportunities and threats (SWOT) is a method to formulate the strategy. The SWOT analysis successfully provides the key factors of the problem. In recent years, the multiple criteria decision making (MCDM) has removed some of the deficiencies. However, the nature of these decisions is usually very complex and using crisp data is not suitable. This paper presents a modification of the Saaty-s scale, in which fuzzy numbers have been used for determining weight values of criteria and alternatives. The method described here takes into account the level of uncertainty of the decision maker. After the application of the AHP method in this way, the values of the functions criteria for each considered alternative are obtained. FA'WOT is a hybrid method combining the well-known SWOT analysis and the Fuzzy AHP. This model is used in determining the development strategy for cybersecurity management bodies.
\end{abstract}

Keywords:

Cybersecurity, organizational structure design, fuzzy logic, multi-criteria decision making, fuzzy AHP.

\section{INTRODUCTION}

Cybersecurity is a major concern of organizations today. More and more organizations rely on cybersecurity to facilitate essential business processes. Cybersecurity covers the steps an organization must take to protect information that can be accessed via vulnerabilities in its networks and systems. However, there are many cybersecurity incidents and failures leading to substantial revenue losses. To solve these difficulties of cybersecurity, organizations must take not only information data and technology, but also managerial and operational aspects into consideration. Every task set for the management must be performed properly and reliably in all environmental conditions. Cybersecurity Management Systems (CSMS) are becoming more and more popular for the organizations that want to improve their cybersecurity levels. The CSMS is powered by 
a set of controls. The effectiveness of the whole CSMS is directly decided by the effectiveness of the implemented cybersecurity in departments [1].

Different departments in an organization have different security responsibilities and thus adopt different controls in CSMS. With different responsibilities in the cybersecurity management, different departments are concerned with different aspects of cybersecurity. The departments with relation to security are Management Board, HR Department, Security Department, Information Protection Department, and Information System Management Department.

Organizational design is specific to every organization, and therefore a unitary organizational structuring cannot be established. Various approaches to organizational design are possible not only due to the differences between companies, but also because certain factors affect different companies in different ways.

In recent years, the methodological approaches of computational modelling and simulation are becoming increasingly popular among organizational researchers. Simulation, unlike mathematical modelling, allows researchers to reflect the natural complexity of organization systems as given. Computational modelling facilitates studies of more complex systems than traditional mathematical approaches.

Computational and mathematical models of organizational design may be found in the papers by Carley [2].

Kujacic and Bojovic [3] proposed a model for selecting the organizational structure using fuzzy multicriteria analysis. The developed fuzzy multi-criteria methodology takes into consideration uncertainty and imprecision of the input data. Researchers in the field of computational organization theory use computational analysis methods to study both humans and organizations as computational entities. Human organizations can be viewed as intrinsically computational, as many of their activities involve sharing and transforming information from one form to another and also because organizational activity is often information-driven [4].

Some design approaches to organizational structure with emphasis on the basic characteristics of each of these models has been presented in the first part of the paper. In the continuation, a model for the selection of optimal variants of organization based on fuzzy logic has been developed starting from the relevant theory approach. Fuzzy AHP is applied in the SWOT analysis (FA'WOT model) to optimize the existing organizational structure of the management bodies of cybersecurity. The choice of organizational models is made using Fuzzy AHP and standard techniques of multi-criterion decision making [5]. The aforementioned model is shown in the following section of the paper.

\section{FA'WOT MODEL}

In the process of designing the organizational structure, certain decisions have to be made. It is worth pointing out that subjective evaluation of certain parameters differ from one decision-maker to another. Quite a convenient approach in quantifying these parameters is the fuzzy set theory introduced by Lotfi Zadeh [6].

Since fuzzification of the AHP method is primarily based on fuzzification of the grading scale, the following part of this paper will present approach to optimization of the dynamic grading scale [7,9]. Fuzzification of the basic AHP method has been done in such a way that triangle fuzzy numbers are used for determining the essential criteria values and fuzzy arithmetic for the whole procedure. Fuzzy numbers are intuitively easy to use when expressing the decision maker's qualitative assessments $[8,9]$.

This way of defining the confidence interval does not take into account the level of uncertainty used for the evaluation of linguistic expressions. The level of uncertainty is represented by the length of the fuzzy number base. In other words, the greater uncertainty in assessment of the linguistic expression the bigger the length of the base (certainty interval) of the fuzzy number [9].

Unlike the above mentioned models, the one represented in this paper takes into account the level of uncertainty which is marked by parameter $\beta$. In this case, the greatest possible uncertainty is described by the value $\beta=0$, while the value $\beta=1$ corresponds the situation in which we are totally sure that the linguistic expression corresponds the given comparison of the optimality criteria. The value of the parameter $\beta$ can be any number within the interval $[0,1]$. In this way, upper and lower limits of the confidence interval of the fuzzy number are chosen randomly for the given value of the parameter $\beta$, so that they are within the limits defined by the expression (1) [9]:

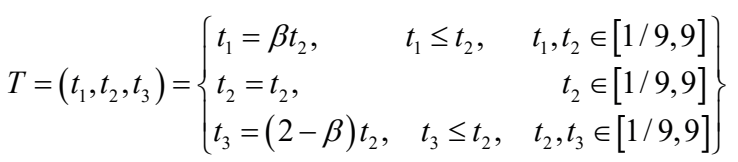


Application of the described procedure implies the realization of Saaty's scale fuzzification (Table 1) where fuzzy number $T=\left(t_{1}, t_{2}, t_{3}\right)=(x \beta, x,(2-\beta) x), x \in[1,9]$ is defined as:

$$
\begin{aligned}
& t_{1}=x \beta= \begin{cases}x \beta, & \forall 1 \leq x \beta \leq x \\
1, & \forall x \beta<1\end{cases} \\
& t_{2}=x, \forall x \in[1,9] \\
& t_{3}=(2-\beta) x= \begin{cases}(2-\beta) x, & \forall x \leq(2-\beta) x \leq 9 \\
9, & \forall(2-\beta) x>9\end{cases}
\end{aligned}
$$

\begin{tabular}{|c|c|c|c|}
\hline \multirow{2}{*}{$\begin{array}{c}\text { Importance } \\
\text { intensity }\end{array}$} & \multirow{2}{*}{ Definition } & \multicolumn{2}{|c|}{ Fuzzified values } \\
\hline & & Fuzzy number & Inversive fuzzy number \\
\hline 1 & $\begin{array}{l}\text { Same } \\
\text { importance }\end{array}$ & $\begin{array}{l}(1,1,1) \text { compared with oneself, } \\
(\beta, 1,2-\beta) \text { in other cases }\end{array}$ & $\begin{array}{l}(1 / \beta, 1,1 /(2-\beta)) \\
\text { in other cases }\end{array}$ \\
\hline 3 & $\begin{array}{l}\text { Weak } \\
\text { dominance }\end{array}$ & $(3 \beta, 3,(2-\beta) 3)$ & $(1(2-\beta) 3,1 / x, 13 \beta)$ \\
\hline 5 & $\begin{array}{l}\begin{array}{l}\text { Strong } \\
\text { dominance }\end{array} \\
\end{array}$ & $(5 \beta, 5,(2-\beta) 5)$ & $(1(2-\beta) 5,1 / 5,15 \beta)$ \\
\hline 7 & $\begin{array}{l}\text { Very strong } \\
\text { dominance }\end{array}$ & $(7 \beta, 7,(2-\beta) 7)$ & $(1(2-\beta) 7,1 / 7,17 \beta)$ \\
\hline 9 & $\begin{array}{l}\text { Absolute } \\
\text { dominance }\end{array}$ & $(9 \beta, 9,(2-\beta) 9)$ & $(1(2-\beta) 9,1 / 9,19 \beta)$ \\
\hline$x=2,4,6$ & Inter-values & $(x \beta, x,(2-\beta) x)$ & $(1(2-\beta) x, 1 / x, 1 \beta x)$ \\
\hline
\end{tabular}

Table 1. Fuzzified Saaty's scale

Inversive fuzzy number

$T^{-1}=\left(1 / t_{1}, 1 / t_{2}, 1 / t_{3}\right)=(1 /(2-\beta) x, 1 / x, 1 / \beta x), x \in[1 / 9,1]$ is defined as:

$1 / t_{1}=1 /(2-\beta) x=\left\{\begin{array}{l}1 /(2-\beta) x, \forall x<1 /(2-\beta) x<1 \\ 1, \forall 1 /(2-\beta) x>1\end{array}\right.$

$1 / t_{2}=1 / x, \forall 1 / x \in[1 / 9,1]$

$1 / t_{3}=1 / \beta x=\left\{\begin{array}{l}1 / \beta x, \quad \forall 1 / 9 \leq 1 / \beta x \leq 1 / x \\ 1 / 9, \quad \forall 1 / \beta x<1 / 9\end{array}\right.$

In this way, the values of criteria functions for every considered alternative are obtained after the application of the AHP method. Certain value of parameter $\beta$ corresponds to values of criteria functions obtained. It is possible to generate various sets of values of the criteria functions for various values of parameter $\beta$ [9].

After defining the parameters of fuzzy Saaty's scale [7-10] conditions have been created to describe the steps for implementing FA'WOT model (Fig.3).

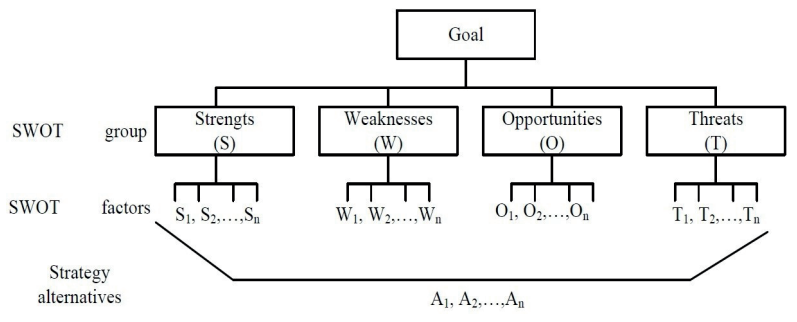

Fig. 1. The hierarchical presentation of FA'WOT analysis

FA'WOT model includes the following steps:

Step 1. Identify SWOT sub-factors and determine the alternative strategies according to the SWOT sub-factors. Determine the importance degrees of the SWOT factors.

Step 2. The Aggregation Principle. This is important for understanding the aggregation process of judgments made at two consecutive hierarchical levels, where criteria and sub-criteria are located. Here criteria and sub-criteria are aggregated by shifting criteria at the sub-criteria level. After the shift, the whole criteria level does not exist anymore. The aggregation principle is illustrated in (Fig. 2).

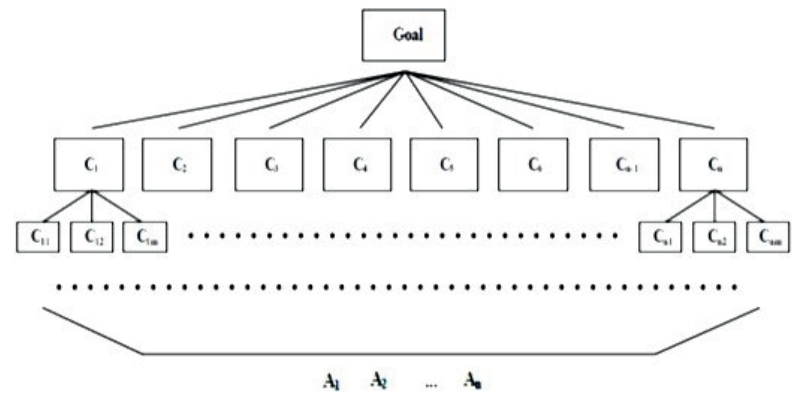

Fig. 2. Aggregation of criteria and sub criteria levels

Step 3. Evaluating Criteria. The ranking procedure starts with the determination of the importance of criteria with respect to the goal [10]. By using a fuzzified scale, a fuzzy reciprocal judgment matrix for criteria is determined as (8) [9]:

$A=\left[\begin{array}{ccccc}\tilde{a}_{11} & \cdots & \tilde{a}_{1 n-m} & \cdots & \tilde{a}_{M} \\ \vdots & & & & \vdots \\ a_{k 1} & \cdots & a_{k n-M} & \cdots & a_{k M} \\ \vdots & & & & \vdots \\ a_{K 1} & \cdots & a_{K n-M} & \cdots & a_{K M}\end{array}\right]$

where $a_{i j}=1$ for all $i=j(i, j=1,2, \ldots, M)$ and $a_{i j}=1 / a_{j i}$. 
By applying the fuzzy synthetic extent, the corresponding weights of criteria can be determined as (9):

$$
w_{i}=\frac{\sum_{j=1}^{M} a_{i j}}{\sum_{k=1}^{M} \sum_{l=1}^{K} a_{k l}}, \sum_{i=1}^{K} w_{i}=1, w_{i} \in[0,1], i=1,2, \ldots M
$$

It should be noted that fuzzy extent (9) could be defined as the result of fuzzy arithmetic, or by using the extension principle. The second is slightly more difficult, but would lead to reduced uncertainty.

Step 4. Evaluating Sub-criteria. For the given criterion $\mathrm{C}_{\mathrm{j}}$, which splits into $k_{\mathrm{j}}$ sub-criteria, it is necessary to determine the relative importance of the sub-criteria with respect to this criterion. After that the fuzzy judgment matrix can be determined as (10) [9]:

$$
A_{j}=\left[\begin{array}{cccc}
a_{11} & a_{12} & \ldots & a_{1 k_{j}} \\
a_{21} & a_{22} & \ldots & a_{2 k_{j}} \\
\ldots & \ldots & \ldots & \ldots \\
a_{k_{j} 1} & a_{k_{j} 2} & \ldots & a_{k_{j} k_{j}}
\end{array}\right]
$$

Final sub-criteria weights are derived through the aggregation of the weights at two consecutive levels [10]. Multiplying sub-criteria weights by respective criterion weight (9) yields (11):

$$
w_{j}^{p}=\left(\frac{\sum_{l=1}^{k_{j}} a_{i l}}{\sum_{i=1}^{k_{j}} \sum_{l=1}^{k_{j}} a_{i l}}\right) \cdot w_{j}, j=1,2, \ldots M ; p=1,2, \ldots, k_{j}
$$

where $w_{j}^{p}$ are the aggregated fuzzy weights of subcriteria. They are entries of the weight vector (11) with the total length $K$.

$$
W=\left(w_{1}^{1}, w_{1}^{2}, \ldots, w_{1}^{k 1}, w_{2}^{1}, w_{2}^{2}, . ., w_{2}^{k 2}, \ldots, w_{j}^{1}, w_{j}^{2}, . ., w_{j}^{k j}, \ldots, w_{M}^{1}, w_{M}^{2}, . ., w_{M}^{k M}\right)
$$

Step 5. Evaluating alternatives. The provided $\mathrm{N}$ alternatives are pairwise compared with respect to each of the K sub-criteria [9]. After obtaining $K$ fuzzy judgment matrices of type (12), the fuzzy extent (13) produces the decision matrix 16.

$W_{k}=\left[\begin{array}{cccc}a_{11} & a_{12} & \ldots & a_{1 N} \\ a_{21} & a_{22} & \ldots & a_{2 N} \\ \ldots & \ldots & \ldots & \ldots \\ a_{N 1} & a_{N 2} & \ldots & a_{N N}\end{array}\right], k=1,2, \ldots, K$
After obtaining K fuzzy judgment matrices of type (12), the fuzzy extent (13) produces the decision matrix (14).

$$
x_{i j}=\frac{\sum_{k=1}^{K} a_{i k}}{\sum_{l=1}^{N} \sum_{m=1}^{K} a_{l m}}, i=1,2, \ldots N ; j=1,2, \ldots, K
$$

$X=\left[\begin{array}{cccc}x_{11} & x_{12} & \ldots & x_{1 N} \\ x_{21} & x_{22} & \ldots & x_{2 N} \\ \ldots & \ldots & \ldots & \ldots \\ x_{N 1} & x_{N 2} & \ldots & x_{N N}\end{array}\right], k=1,2, \ldots, K$

In the decision matrix $X, x_{i j}$ represents the resultant fuzzy performance assessment of the alternative $A_{i}(i=1,2, \ldots, N)$ with respect to the $j$ th sub-criterion $(j=1,2, \ldots, K)$.

Step 6. Performance matrix. An overall performance of each alternative across all sub criteria may be represented by the fuzzy performance matrix (16).

$Z=\left[\begin{array}{cccc}x_{11} w_{1} & x_{12} w_{2} & \ldots & x_{1 N} w_{K} \\ x_{21} w_{1} & x_{22} w_{2} & \ldots & x_{2 N} w_{K} \\ \ldots & \ldots & \ldots & \ldots \\ x_{N 1} w_{1} & x_{N 2} w_{2} & \ldots & x_{N N} w_{K}\end{array}\right]$

Step 7. Final assessments and synthesis. The final alternative performance weights with respect to the overall goal are calculated by the summation of elements in the rows of the performance matrix (15) to obtain equation (17).

$F_{i}=\sum_{j=1}^{K} x_{i j} w_{j}, \quad i=1,2, \ldots, N$

To finally rank the alternatives, the prioritization of aggregated assessments is required. Since each $F_{i}$ is a triangular fuzzy number, it is necessary to apply the method of ranking triangular fuzzy numbers. For the given triangular fuzzy number $\mathrm{A}=\left(a_{1}, a_{2}, a_{3}\right)$ the total integral value is defined as [11] (18):

$I_{T}^{\lambda}(A)=\left[\lambda a_{3}+a_{2}+(1-\lambda) a_{1}\right] \cdot 2^{-1}, \quad \lambda \in[0,1]$

$\lambda$ represents an optimism index which expresses the decision maker's attitude towards risk. A larger value of $\lambda$ indicates a higher degree of optimism. In practical 
applications, values $0,0.5$ and 1 are used respectively to represent the pessimistic, moderate and optimistic views of the decision maker.

The final ranking of alternatives means adopting a certain level $\lambda$ of optimism of the decision-maker, then applying equation (19) on fuzzy numbers equation (17), and finally ranking alternatives regarding values obtained for $I_{T}^{\lambda}(F), i=1,2, \ldots, N$. [9] The best alternative from the set is represented as:

$$
f_{F_{i}}=\max \left(f_{F_{i}}\right), i=1,2, \ldots, A
$$

\section{APPLICATION OF FA' WOT MODEL IN ORGANIZATIONAL DESIGN OF THE CYBERSECURITY MANAGEMENT BODIES}

Enterprises are moving their business processes towards digitization, mobility, big data analytics, web collaboration and cloud services. Most of them usually do not consider enterprise IT security strategy a mandatory factor and lack adequate cybersecurity organizational structure.

This, however, makes up for a poor organizational strategy that leads nowhere. Businesses should recognize enterprise cybersecurity as their priority. Personal data, digital intellectual property, enterprise cyber infrastructure and even business-critical apps can be compromised via network attacks, break-ins, inadvertent security lapses, vulnerable web services etc.

Designing a CSMS has a large influence on the creation, adaptation, existence and quality of the system operation. No organizational system within the cybersecurity support can operate independently of its management subsystem responsible for issuing commands for the desired "behavior" of the system, while the actual behavior can deviate from the desired. SWOT analysis is used to manage the total organization, the overall pattern of structural components and arrangement. Through the analysis of the internal and external factors, which affect organizational changes and organizational structuring, four different types of cybersecurity organizations in enterprises were obtained. Applying modified AHP method, evaluation of the suggested types was performed and the most affordable option of the organizational structure was chosen. The decision hierarchy of FA'WOT model is defined as follows:

1. Goal: to identify the best alternative organizational

2. Criteria (Level 1) and sub-criteria (Level 2):

\section{$\underline{\text { S. Strengths }}$}

$\mathrm{S}_{1}$ : Simple structure

$\mathrm{S}_{2}$ : The minimum number of hierarchical levels

$\mathrm{S}_{3}$ : Functional grouping of working processes

$S_{4}$ : Quick transfer of information

$\mathrm{S}_{5}$ : Avoidance of resources duplication within functions of the cybersecurity support

$\mathrm{S}_{6}$ : Simple coordination within the cybersecurity Support functioning

$\mathrm{S}_{7}$ : Possibility of preferment in career (career development)

$\mathrm{S}_{8}$ : The ability to track personnel development and motivation

$S_{9}$ : Low probability of dismissal

\section{$\underline{\text { W. Weaknesses }}$}

$\mathrm{W}_{1}$ : Low specialization toward work processes

$\mathrm{W}_{2}$ : Need for better coordination between the cybersecurity support functions

$\mathrm{W}_{3}$ : Poor communication between the cybersecurity support functions

$\mathrm{W}_{4}$ : Danger of conflict in defining priorities

$\mathrm{W}_{5}$ : Focusing on section (departmental) problems

$\mathrm{W}_{6}$ : Difficult co-ordination between the cybersecurity support functions when making plans

$\mathrm{W}_{7}$ : Development of managers (executives) specialized in specific areas

$\mathrm{W}_{8}$ : Close monitoring of organizational goals

$\mathrm{W}_{9}$ : The risk of accumulation of decisions at the top of the hierarchy

\section{O. Opportunities}

$\mathrm{O}_{1}$ : Effective in a stable environment

$\mathrm{O}_{2}$ : Strong management team

$\mathrm{O}_{3}$ : Training of existing and introduction of new personnel in the administrative structure of the cybersecurity support

$\mathrm{O}_{4}$ : Capability for organization of management processes cybersecurity bodies

$\mathrm{O}_{5}$ : Introduction of up-to-date informational technology into the process of the cybersecurity support management

$\mathrm{O}_{6}$ : Automated managing of cybersecurity support processes

\section{$\underline{\text { T. Treats }}$}

$\mathrm{T}_{1}$ : Slow adaptation to changes in the environment 
$\mathrm{T}_{2}$ : Weak innovative capabilities

$\mathrm{T}_{3}$ : Changing of positions of the expert personnel by the cybersecurity support functions

$\mathrm{T}_{4}$ : Introduction of "outsourcing" technology in some of the functions of cybersecurity support

$\mathrm{T}_{5}$ : Requirements for rapid adaptation and response to social changes

3. Types of the organizational structure of administrative bodies (Level 3):

Alternative 1. Represents an existing organization of cybersecurity management bodies in an enterprise.

Alternative 2. Represents a modification of the existing organization of cybersecurity management bodies in an enterprise. Application of the cybersecurity approach and cybersecurity organization upon a functional principle is the basis for development of II type. This type is based on the internal division of labor, specialization and differentiation of organizational units and holders of management. Grouping of individual functions, processes and activities was done by the following principles: the executive principle (grouping of individual processes executive personnel into a single function, which creates a functional organization), the property principle (grouping according to proprietors for whom a job is performed or who perform the functions, processes and activities), the rank principle (grouping according to priority and executive level), according to the phases (grouping according to phases of planning, implementation and control), by purpose principle (grouping according to organizing administrative processes feasibility and functions of a particular business in certain organizational units). Functional structure model is based on the functional division of labor, it is oriented towards the labor process, and based also on functional groupings of units and the optimization of employee numbers.

Alternative 3.Represents the organization of administrative bodies upon cybersecurity processes. The governing process (management) within an organization consists of five basic sub-processes or phases: planning, organizing, leadership, personnel management and controlling. All stages are interrelated and interdependent and make a continuous process of managing the cybersecurity support. These stages, or organizational units are attached to each other, connected and interrelated. After the process of planning, the process of organization continues, associated with the previous one by direct channels and direct feedback. The advantage of such type of organizing in support of administrative authority is the fact that specialized personnel is grouped by the same process or governing stage and they are oriented towards the realization of the process. The disadvantage is reflected in the requirement for better coordination of the entire work, preventing problems in one process causing problems in the whole business.

Alternative 4. Represents the organization of the administrative organs of cybersecurity by processes and by functions. In this type of the organizational structure, positive sides of functional organization and organizations designed upon managing processes are taken into consideration.

To determine relative importance of the evaluation criteria Strengths, Weaknesses, Opportunities and Treats, they were pairwise compared with respect to the goal by using the fuzzified scale given in Table 1 [7-10]. Linguistically expressed preferences among criteria have been used to create a judgment matrix A as given by (8):

$A_{S W O T}==\underset{\mathrm{S}}{\mathrm{W}}\left[\begin{array}{cccc}\mathrm{S} & \mathrm{W} & 0 & \mathrm{~T} \\ \mathrm{~T} & \tilde{3} & \tilde{3} & \tilde{3} \\ \mathrm{~T} & \tilde{1} & \tilde{2} & \tilde{3} \\ \mathrm{~T} & \tilde{3}^{-1} \tilde{2}^{-1} & \tilde{1} & \tilde{6} \\ \tilde{2}^{-1} \tilde{2}^{-1} & \tilde{\sigma}^{-1} \tilde{1}\end{array}\right] \Rightarrow W_{c_{2}}=\left[\begin{array}{c}W_{S} \\ W_{W} \\ W_{o} \\ W_{T}\end{array}\right]=\left[\begin{array}{c}(0.259,0.370,0.481) \\ (0.076,0.096,0.125) \\ (0.268,0.384,0.499) \\ (0.105,0.151,0.196)\end{array}\right]$

The weighting vector w of criteria matrix $A_{S W O T}$ was determined by applying (9). Each entry of this vector is the sum of elements in the related row of matrix $A_{\text {SWOT }}$ divided by the sum of all its elements. For example:

$$
\begin{aligned}
& W_{s} \\
& =\frac{\tilde{1}+\tilde{3}+\tilde{3}+\tilde{2}}{\tilde{1}+\tilde{3}+\tilde{3}+\tilde{2}+\tilde{3}^{-1}+\tilde{1}+\tilde{2}^{-1}+\tilde{2}^{-1}+\tilde{3}^{-1}+\tilde{2}+\tilde{1}+\tilde{6}+\tilde{2}^{-1}+\tilde{2}+\tilde{6}^{-1}+\tilde{1}} \\
& =(0.259,0.370,0.481) \\
& W_{\text {SWOT }}=\left[\begin{array}{l}
W_{S} \\
W_{W} \\
W_{o} \\
W_{T}
\end{array}\right]=\left[\begin{array}{l}
(0.140,0.200,0.259) \\
(0.067,0.096,0.125) \\
(0.268,0.384,0.499) \\
(0.105,0.151,0.196)
\end{array}\right]
\end{aligned}
$$

In the next step, through the use of fuzzy pairwise comparisons, the judgment matrices (10) for sub-criteria related to respective criteria were obtained. Related sub-criteria weighting vectors were calculated as defined by (9):

$W_{S}=\left[\begin{array}{l}W_{s_{1}} \\ W_{s_{2}} \\ W_{s_{3}} \\ W_{s_{4}} \\ W_{s_{5}} \\ W_{s_{6}} \\ W_{s_{7}} \\ W_{s_{8}} \\ W_{s_{9}}\end{array}\right]=\left[\begin{array}{l}(0.110,0.157,0.204) \\ (0.041,0.059,0.077) \\ (0.016,0.023,0.030) \\ (0.120,0.171,0.222) \\ (0.063,0.090,0.117) \\ (0.076,0.108,0.141) \\ (0.060,0.085,0.111) \\ (0.121,0.173,0.225)\end{array}\right]$ 
$W_{w}=\left[\begin{array}{l}W_{w_{1}} \\ W_{w_{2}} \\ W_{w_{3}} \\ W_{w_{4}} \\ W_{w_{5}} \\ W_{w_{6}} \\ W_{w_{7}} \\ W_{w_{8}} \\ W_{w_{9}}\end{array}\right]=\left[\begin{array}{l}(0.177,0.252,0.328) \\ (0.074,0.106,0.138) \\ (0.046,0.065,0.085) \\ (0.106,0.151,0.195) \\ (0.030,0.043,0.056) \\ (0.062,0.088,0.115) \\ (0.103,0.147,0.191) \\ (0.080,0.144,0.148)\end{array}\right]$

$W_{o}=\left[\begin{array}{l}W_{o_{1}} \\ W_{o_{2}} \\ W_{o_{3}} \\ W_{o_{4}} \\ W_{o_{5}} \\ W_{o_{6}}\end{array}\right]=\left[\begin{array}{l}(0.230,0.328,0.427) \\ (0.251,0.359,0.467) \\ (0.095,0.135,0.176) \\ (0.024,0.034,0.044) \\ (0.039,0.055,0.072) \\ (0.062,0.088,0.115)\end{array}\right]$

$W_{T}=\left[\begin{array}{l}W_{T_{1}} \\ W_{T_{2}} \\ W_{T_{3}} \\ W_{T_{4}} \\ W_{T_{5}}\end{array}\right]=\left[\begin{array}{l}(0.140,0.200,0.259) \\ (0.071,0.101,0.131) \\ (0.357,0.510,0.664) \\ (0.026,0.038,0.049) \\ (0.106,0.151,0.197)\end{array}\right]$

By fuzzy multiplication of the related sub-criteria weighting vectors and criteria weights, as given in (11), the aggregated weights of the sub-criteria were obtained with respect to the goal. For example:

$W_{S}^{\prime}=W_{s_{i}} \cdot W_{s}=\left[\begin{array}{l}W_{s} \cdot W_{s_{1}} \\ W_{s} \cdot W_{s_{2}} \\ W_{s} \cdot W_{s_{3}} \\ W_{s} \cdot W_{s_{4}} \\ W_{s} \cdot W_{s_{5}} \\ W_{s} \cdot W_{s_{6}} \\ W_{s} \cdot W_{s_{7}} \\ W_{s} \cdot W_{s_{8}} \\ W_{s} \cdot W_{s_{9}}\end{array}\right]=\left[\begin{array}{l}(0.140,0.200,0.259) \cdot(0.110,0.157,0.204) \\ (0.140,0.200,0.259) \cdot(0.041,0.059,0.077) \\ (0.140,0.200,0.259) \cdot(0.016,0.023,0.030) \\ (0.140,0.200,0.259) \cdot(0.120,0.171,0.222) \\ (0.140,0.200,0.259) \cdot(0.063,0.090,0.117) \\ (0.140,0.200,0.259) \cdot(0.076,0.108,0.141) \\ (0.140,0.200,0.259) \cdot(0.060,0.085,0.111) \\ (0.140,0.200,0.259) \cdot(0.093,0.133,0.173) \\ (0.140,0.200,0.259) \cdot(0.121,0.173,0.225)\end{array}\right]$

$$
\begin{array}{cccc}
A_{1} & A_{2} & A_{3} & A_{4} \\
(0.0 .030,0.32,036) & (0.36,0.32,0.30) & (0.16,0.19,0.21) & (0.12,0.16,0.19
\end{array}
$$

$\left[\begin{array}{llll}(0.30,0.32,0.36) & (0.36,0.32,0.30) & (0.16,0.19,0.21) & (0.12,0.16,0.19)\end{array}\right]\left[\begin{array}{l}0.33 \\ 0.23\end{array}\right]$ $F=\left[\begin{array}{llll}(0.20,0.21,0.23) & (0.24,0.26,0.23) & (0.19,0.21,0.23) & (0.30,0.33,0.35)\end{array}\right] \times\left[\begin{array}{l}0.29 \\ 0.20\end{array}\right.$ $\left[\begin{array}{llll}(0.07,0.08,0.11) & (0.29,0.31,0.32) & (0.26,0.27,0.29) & (0.26,0.27,0.29)\end{array}\right] \times\left[\begin{array}{l}0.29 \\ 0.17 \\ 0.17\end{array}\right]$

$\begin{array}{llll}(0.14,0.14,0.17) & (0.30,0.31,0.32) & (0.21,0.23,0.25) & (0.29,0.31,0.34)\end{array}$

Table 2. Ranking of alternatives

\begin{tabular}{ccccc}
\hline \multicolumn{5}{c}{ Index of optimism } \\
\hline $\begin{array}{c}\text { Decision } \\
\text { alternative }\end{array}$ & $\begin{array}{c}\boldsymbol{\lambda}=\mathbf{0 . 0} \\
\text { (pessimistic) }\end{array}$ & $\begin{array}{c}\boldsymbol{\lambda}=\mathbf{0 . 5} \\
\text { (moderate) }\end{array}$ & $\begin{array}{c}\boldsymbol{\lambda}=\mathbf{1 . 0} \\
\text { (optimistic) }\end{array}$ & $\begin{array}{c}\text { Final } \\
\text { rank }\end{array}$ \\
\hline Alternative 1 & 0.205 & 0.212 & 0.220 & 4 \\
\hline Alternative 2 & 0.285 & 0.295 & 0.305 & 1 \\
\hline Alternative 3 & 0.210 & 0.220 & 0.230 & 3 \\
\hline Alternative 4 & 0.245 & 0.255 & 0.265 & 2 \\
\hline
\end{tabular}

The assessment of the alternatives has been performed using relations (13), (14) and (15). The final alternative performance weights, with respect to the overall goal, have been calculated by equation (17) as shown:

$V=\left[\begin{array}{l}V_{1} \\ V_{2} \\ V_{3} \\ V_{4}\end{array}\right]=\left[\begin{array}{l}(0.20,0.21,0.23) \\ (0.28,0.29,0.32) \\ (0.20,0.22,0.24) \\ (0.22,0.26,0.27)\end{array}\right]$

For the typical values of $\lambda$ that express the decisionmaker's attitude toward risk, the final ranking of alternatives is obtained by applying equation (18). The normalized values presented in Table 2 show that Alternative 2 is the best. It is followed by Alternative 4 , Alternative 3 and Alternative 1 respectively, regardless of the decision-maker's level of optimism.

By using the center of gravity method to defuzzify the $V$ values given above, the final weights of alternatives obtained after normalization were: 0.213 (Alternative 1), 0.297 (Alternative 2), 0.220 (Alternative 3) and 0.253 (Alternative 4). Obviously, the final ranking is equal to the previous one.

\section{DISCUSSION AND CONCLUSION}

Organization is not a sum of mechanical parts, rather an "organic whole" with a purpose and mission. In the process of designing the organizational structure it is necessary, having defining the objectives and design criteria, to analyze the state of the organization.

In addition to organizations operating in an uncertain environment, there is a degree of uncertainty and imprecision of criteria used in the process of organizational design. Fuzzy multi-criteria approach developed in this paper allows the quantification of these criteria and selection of the best alternative out of the proposed organizational models. For the academic audience, we present effective and contemporary modeling using mathematical solution for application in the area of cybersecurity. The presented model enables the evaluation of the proposed options of organizational structure, regardless of the number of optimality criteria and subcriteria. The model allows for the choice of best alternative from a set described ones using K optimality criteria and sub-criteria.

Application of the given model is shown on the example of designing the organizational structure of the management of the enterprise cybersecurity. 
The complex environment, in which these management bodies act, does not tolerate organizational improvisation, rather requires a planned and methodological organizational approach.

\section{REFERENCES}

[1] Hong-li Liu, Ying-ju Zhu, "Measuring Effectiveness of Information Security Management", International Symposium on Computer Network and Multimedia Technology, 2009.

[2] K. M.. Carley, "Computational and mathematical organization theory: perspective and directions", Computational and mathematical organization theory, 1(1),1995 pp 39-56

[3] M. Kujacić, N. Bojović, "Organizational design of post corporation structure using fuzzy multicriteria decision making". Computational and mathematical organization theory, 9, 2003, p. 5-18.

[4] D. Pamučar, B. Djorović, " Fuzzy logic applied to organizational design of the administrative management", Metalurgia international, 17 (5), 2012 pp. 87-96.

[5] B. Djorovic, D. Pamučar, Projektovanje organizaconih struktura: metode i modeli, Medija centar „Odbrana“, Beograd 2016., p. 133-219
[6] Zadeh, L. A., "Fuzzy sets", Inf control 8, 1965 p.338-353.

[7] D. Božanić, S. Karović, D. Pamučar, "Fazifikacija Saaty-jeve skale primenom trouglastog fuzzy broja sa promenljivim intervalom poverenja", Zbornik radova, SYM-OP-IS, ISBN 978-86-80593-55-5, 2015p. 420-424.

[8] D. Bojanić, M. Kovač, M. Bojanic, V. Ristic, “Multicriteria decision-making in a defensive operation of the guided anti-tank missile battery: An example of the hybrid model fuzzy AHP - MABAC". Decision Making: Applications in Management and Engineering, 1(1), Vol. 1, Number 1, 2018, ISSN: 2560-6018 p.51-66.

[9] Pamučar, D., Ćirović, G., i Sekulović, D. “Development of an integrated transport system in distribution centres: a FA'WOT analysis", Tehnički vjesnik, 22(3), p.649-658. doi: https://doi.org/10.17559/TV20140205170207

[10] D. Pamučar, G. Ćirović, D. Sekulović, A. Ilić, “A new fuzzy mathematical model for multi criteria decision making: An application of fuzzy mathematical model in a SWOT analysis" Scientific Research and Essays, 2011, 6(25), 5374-5386..

[11] B. Djorovic B., D. Pamucar, N. Dimitrijević, “Application of fuzzy logic and fuzzy sets for quantification of input and output parametres in DEA analisys", Metalurgia international, 17 (5) 2012 p. 78-86. 